\title{
DETERMINANTES SOCIAIS DA LEISHMANIOSE VISCERAL NO NORTE DE MINAS GERAIS ${ }^{1}$
}

\author{
Chrystian Soares Mendes ${ }^{2}$ \\ Luckas Sabioni Lopes ${ }^{3}$ \\ Silvia Harumi Toyoshima ${ }^{4}$
}

RESUMO: A Leishmaniose Visceral (LV) é uma zoonose típica de regiões tropicais, transmitida pelo inseto fêmea da espécie Lutzomya longipalpis. O Brasil é um dos países que mais tem sofrido com tal endemia, dadas as grandes mudanças no ambiente natural do mosquito, advindas do crescimento exacerbado das cidades e desmatamentos da mata ciliar. No país, o Estado de Minas Gerais é o terceiro em número de casos de LV, e a Mesorregião Norte é a que apresenta o maior número de incidências. O objetivo deste estudo foi verificar a influência de indicadores socioeconômicos sobre a LV nessa região, bem como criar e discriminar grupos de municípios segundo a incidência desta zoonose no período compreendido entre 1998 e 2008, por meio de técnicas estatísticas multivariadas. Percebeu-se que a LV não se reduziu no decorrer dos anos, ao contrário, ela se elevou. O Município de Matias Cardoso foi o que apresentou maior elevação no período (550\%). No que concerne à discriminação dos agrupamentos, foi observada forte influência da urbanização e do saneamento básico no combate ao vetor. A educação continuada e a adoção de políticas públicas que visem à conscientização da população também são necessárias para o controle adequado da doença.

Palavras-chave: Leishmaniose Visceral; Determinantes Sociais; Norte de Minas; Análise Multivariada.

Recebido em: 08/02/2011. Aceito em: 11/04/11.

Doutorando em Economia Aplicada - Universidade Federal de Viçosa-MG. E-mail: chrystian.mendes@ufv.br.

Doutorando em Economia Aplicada - Universidade Federal de Viçosa-MG. E-mail: luckas.lopes@ufv.br.

4 Doutorado em Economia - Universidade Estadual de Campinas - SP (1997). Pós-Doctor - University of Illinois - EUA (2006). Profa. Associada do Departamento de Economia da Universidade Federal de ViçosaMG. E-mail: htsilva@ufv.br. 


\begin{abstract}
Visceral Leishmaniasis (VL) is a typical tropical zoonosis, transmitted by the female insect of the genus Lutzomyia longipalpis. Brazil is one of the countries that have suffered with this disease, given the great changes in the natural environment of the mosquito, due to the growth of cities and exacerbated deforestation of riparian forest. In the country, the State of Minas Gerais is the third in numbers of cases of LV, where the North Mesorregion shows the great number of incidences. Therefore, the objective of this study was to evaluate the influence of LV's socio-economic indicators in this region. It created and discriminated municipalities' groups according to the incidence of this zoonosis in the period 1998 to 2008 using the multivariate statistics. It was noticed that LV was not reduced over the years; on the contrary it increased. The Municipality of Matias Cardoso had the largest increase in this period (550\%). Regarding the discrimination of the groups, there was the strong influence of urbanization and the importance of sanitation in combating the vector. The education and the public policies aimed at awareness of the population is also necessary to have adequate control of the disease.

Key-words: Visceral Leishmaniasis; Social Determinants; North Minas, Multivariate Analysis.
\end{abstract}

\title{
1. Introdução
}

A Leishmaniose Visceral (LV) é uma zoonose típica das regiões tropicais, causada por um protozoário do gênero Leishmania, transmitida pelo inseto fêmea da espécie Lutzomya longipalpis, comumente chamado de "flebótomo". Seu quadro clínico em humanos varia de febre e anemia até manifestações hemorrágicas, aumento do volume dos linfonodos, perda de peso ou mesmo a morte (CHAVES, 1996; MIRANDA, 2008).

Tem-se verificado, atualmente, uma rápida disseminação da LV por todo o mundo, com registros de casos na Ásia, Europa, Oriente Médio, África e Américas, onde também é denominada Leishmaniose Visceral Americana (LVA), ou calazar neotropical. Na América Latina, a doença já foi observada em pelo menos 12 países, entretanto, $90 \%$ dos casos ocorrem no Brasil, especialmente na Região Nordeste. O registro do primeiro caso dessa doença no país data de 1913, quando Migone o descreveu com material de necropsia de um paciente oriundo da cidade de Boa Esperança, Mato Grosso (ALENCAR et al., 1991). 
Desde então, esta endemia tem se disseminado por várias áreas do território brasileiro. Em Minas Gerais, uma região de grande concentração de casos é a Norte ${ }^{5}$, foco deste trabalho, abrangendo oitenta e nove municípios, tendo como polos as cidades de Montes Claros, Janaúba, Januária, Pirapora, entre outras. Como ilustração, cabe ressaltar que, entre os anos de 2001 e 2005, esta região representou 45\% dos casos de LV do Estado e, apenas o município de Montes Claros, $17 \%$.

Em razão da gravidade desta doença, existem diversos estudos analisando-a no Brasil, como Tavares e Tavares (1999), Pedrosa e Rocha (2004) e Gontijo e Melo (2004), por exemplo. Mais especificamente, sobre o caso do Norte de Minas, podem-se citar os trabalhos de Monteiro et al. (2005), que analisaram um inquérito sorológico canino da LV no período de setembro de 2002 a agosto de 2003 e constataram a prevalência da LV canina na região; Canela et al. (2004), que traçaram um perfil do diagnóstico de LV em pacientes adultos hospitalizados no Hospital Universitário Clemente Faria, em Montes Claros; e Sousa et al. (2008), que avaliaram os aspectos epidemiológicos da doença também na cidade de Montes Claros.

Contudo, há ainda uma lacuna no que diz respeito à análise dos condicionantes sociais da LV na região do Norte de Minas Gerais. Nesse sentido, a principal contribuição desta pesquisa foi avançar nos estudos sobre a endemia, procurando associar sua incidência a aspectos socioeconômicos chave das localidades analisadas, tendo como base a teoria dos "Determinantes Sociais da Saúde" (DAHLGREN e WHITEHEAD, 1991). Para tanto, analisou-se a evolução espacial dos casos de LV ao longo dos anos de 1998 a 2008 e criaram-se agrupamentos municipais homogêneos quanto à incidência desta doença, a fỉm de verificar os principais indicadores socioeconômicos que discriminam a maior ou menor incidência da doença nesta região de Minas Gerais.

5 Mesorregião Norte de Minas. 
Pretende-se, assim, fornecer subsídios para adoção de políticas públicas mais eficazes, isto é, que atuem mais diretamente sobre os principais determinantes socioeconômicos da doença na região em foco. A principal hipótese assumida é que um melhor controle da LV no Norte de Minas Gerais e uma política de saúde pública de elevada importância para os moradores desta localidade passam por medidas educacionais e sociais em geral, e não apenas por ações de saúde propriamente ditas.

\section{Metodologia}

\subsection{Análise de Clusters}

A análise de clusters neste artigo foi realizada com o intuito de construir agrupamentos de municípios com taxa similar de incidência da LV no período. De acordo com Mingoti (2007), essa metodologia tem como objetivo dividir os elementos da amostra, ou população, em grupos de forma que os elementos similares pertençam a um mesmo grupo. Possoli (1984) argumenta que para a construção dos agrupamentos é necessário seguir critérios de similaridade, ou dissimilaridades. Para tanto, normalmente são utilizadas medidas como a distância euclidiana de Mahalanobis ou de Minkoswski, entre outras. Procura-se, assim, agrupar as observações de uma amostra através de algoritmos que têm o intuito de maximizar as diferenças entre os grupos formados, sendo os métodos hierárquicos e não hierárquicos os mais utilizados para tal.

Neste estudo, adotou-se o método hierárquico de Ward, pelo fato de este método propor um agrupamento fundamentado na mudança de variação entre os grupos e dentro dos grupos que vão sendo formados e de ser um método de variância mínima, sob algumas restrições se assemelhando ao de máxima verossimilhança (MINGOTI, 2007).

A análise de agrupamento envolve ainda algumas decisões subjetivas, como o número ótimo de agrupamentos (FERNAU e SAMSON, 1990). 
Para a determinação do número de grupos a serem considerados, não há critério preestabelecido. Neste trabalho, procurou-se uma divisão que refletisse a realidade da distribuição espacial dos indicadores de LV no Norte de Minas; entretanto, também se utilizou como critério uma divisão de municípios que se ajustasse adequadamente ao modelo de análise discriminante realizado, pois, conforme indica Mingote (2007), a análise discriminatória é uma forma de validar a análise de clusters prévia.

\subsection{Análise das variáveis discriminantes dos agrupamentos de municípios quanto à incidência de Leishmaniose Visceral}

A análise discriminante é uma técnica que pode ser utilizada para a classificação de elementos de uma amostra em grupos predeterminados (MINGOTE, 2007). Ela procura criar uma regra matemática, em geral uma função linear de um conjunto de variáveis de interesse, que auxilia na classificação das observações em grupos, ou populações, conhecidas previamente. No caso deste artigo, o número de agrupamentos foi definido na análise anterior de clusters.

O desenvolvimento metodológico da análise discriminante segue o proposto por Mingote (2007). Seja $f_{i}(x)$ a função densidade de probabilidade da população $i, \operatorname{com} i$ definido por $i=1, . ., n$. Para que a regra de discriminação calculada minimize os erros de classificação, ela deve destinar um conjunto de informações $x$, pertencentes a algum elemento amostral, à população (ou grupo) em que se tenha obtido o maior valor para a função densidade de probabilidade, isto é, classificase o elemento amostral naquela população $k$, tal que:

$$
f_{k}(x)=\operatorname{máx}\left\{f_{i}(x), i=1, \ldots, n\right\} .
$$

Em particular, caso o vetor aleatório $x$ em cada população tenha distribuição normal multivariada, esta regra é equivalente a classificar o elemento, utilizando a seguinte equação: 
$d_{k}^{Q}(x)=\operatorname{máx}\left\{d_{1}^{Q}(x), \ldots, d_{n}^{Q}(x)\right\}$,

em que os coeficientes quadráticos $d_{i}^{Q}$ são, na prática, estimados por:

$d_{i}^{Q}(x)=-1 / 2 \ln \left[\left|S_{i}\right|-1 / 2\left(x-\bar{x}_{i}\right) S_{i}^{-1}\left(x-\bar{x}_{i}\right)\right]$,

com $\left(\bar{x}_{i}, S_{i}\right)$ representando, respectivamente, o vetor de médias e a matriz de covariâncias amostrais de cada uma das populações.

A análise da qualidade de ajuste da função discriminante é feita pela comparação das médias populacionais dos escores discriminantes pelo teste de Wilks (ANDERSON, 2003). Outro modo de analisar a qualidade da função discriminante é através da probabilidade de erros de classificação incorreta, que ocorre quando um elemento amostral pertencente à população $j$, por exemplo, é considerado pela regra proveniente de outra população, $k, j \neq k$. As probabilidades de ocorrência destes erros são estimadas por:

$\hat{p}(k / j)=n_{j k} / n_{j}$,

em que $n_{j k}$ é o número de elementos da população $j$ classificados incorretamente pela regra como provenientes da população $k$.

Outra consideração a ser feita a respeito da análise discriminante efetuada é que ela foi estimada por meio do método passo a passo (stepwise), que seleciona em cada etapa a variável mais importante na estimação. Os níveis de significância para entrada e saída de variáveis neste método podem ser mais flexíveis. Serão utilizados, aqui, os níveis propostos por Johnson (1998), quais sejam: 25\% para entrada e 50\% para saída. 


\subsection{Fonte e tratamento dos dados}

Inicialmente, numa primeira exploração da fonte de dados utilizada neste artigo, utilizou-se uma metodologia de análise no que diz respeito à definição das áreas críticas de transmissão da LV baseada nas diretrizes definidas pelo Programa de Controle da Leishmaniose Visceral, do Ministério da Saúde (PCLV, MINISTÉRIO DA SAÚDE, 2003). Seu principal objetivo é estratificar os municípios em dez grupos de igual frequência (decis) quanto à incidência média da doença em períodos de cinco anos. A partir disso, os municípios são classificados em três classes de transmissão de LV, utilizando como critério de ponto de corte o "percentil 90 (P90)" ou, equivalentemente, o nono decil da média dos casos. Assim, os municípios abaixo do P90, ou seja, com média de casos maior que zero e menor que o limite inferior de P90, são rotulados de transmissão esporádica. Os municípios que constituem o P90 são classificados de transmissão moderada, e aqueles que estão acima do limite superior do P90 são considerados de transmissão intensa. Em seguida, procedeu-se à análise de clusters de casos de LV, com a caracterização dos grupos obtidos e, por fim, à análise discriminante, em que se utilizou o número de grupos encontrados na análise de agrupamentos anteriormente.

As variáveis selecionadas para análise foram pautadas no terceiro nível de ação do modelo de determinantes sociais de Dahlgren e Whitehead (1991), pretendendo fornecer evidências dos instrumentos de política que podem levar a resultados mais significativos no controle da $L V$ na região Norte de Minas. A listagem completa dos indicadores considerados é feita na Tabela 1.A do anexo. A seleção das variáveis procurou evidenciar diversas características socioeconômicas, relacionadas ao nível educacional, à renda municipal, à disponibilidade de serviços de saúde, à infraestrutura sanitária, às características demográficas da população e aos recursos públicos gastos que podem influenciar, em maior ou menor grau, na transmissão da doença, de acordo com o que é indicado pela literatura na área. 
Os dados sobre a incidência de LV deste artigo foram obtidos no DATASUS, base digital de informações disponibilizada pelo Ministério da Saúde e são referentes aos anos de 1998 a 2008. Eles foram padronizados, dentro de cada município, em casos por cem mil habitantes, segundo o local de residência do doente. Os indicadores socioeconômicos foram coletados na base de dados da Fundação João Pinheiro (FJP, 2009), para o ano de 2004, e no Instituto Brasileiro de Geografia e Estatística, IBGE, para o ano de 2000.

\section{Resultados e discussão}

\subsection{Dinâmica temporal da Leishmaniose Visceral}

Nesta subseção, são descritos os resultados da análise da dinâmica espaço/temporal da Leishmaniose Visceral entre os anos de 1998/2002 e 2003/2008 no Norte de Minas Gerais. Com respeito ao primeiro período de tempo, 45 municípios apresentaram casos de LV, correspondendo a $51 \%$ deles na região. A média da incidência neste período é 13 quando são consideradas todas as localidades, ascendendo a 25 quando são incluídos apenas os municípios com casos da endemia. O coeficiente de variação da média $(\mathrm{CV})$ para essas amostras diferentes também variou

Amostra $\mathrm{n}=89$ $n=45$ muito, de $135 \%$ para $67 \%$. Isso mostra que a incidência de LV nos 45 municípios que apresentaram casos é mais homogênea que aquela obtida no total das municipalidades. A Tabela 1 resume essas estatísticas descritivas.

Tabela 1 - Estatísticas descritivas da incidência de LV no período de 1998/2002

Fonte: Resultados da pesquisa. 
É interessante neste momento subdividir os municípios que apresentaram casos de LV em percentis, segundo a metodologia da Secretaria de Vigilância Sanitária. A Tabela 2 resume os principais resultados desta estratificação. Vê-se que, entre 1998 e 2002, 50\% dos municípios com casos obtiveram 3,9 ou menos internações. Adicionalmente, 36 localidades foram classificadas como de transmissão esporádica. Isto equivale a $80 \%$ dos municípios com casos e $41 \%$ do total deles. Apesar de serem áreas consideradas de transmissão esporádica, tais localidades apresentaram alta incidência de LV quando comparadas com a média nacional no mesmo período, isto é, o valor do limite superior destas regiões é maior que o valor do P90 nacional, de 4,4 (MINISTÉRIO da SAÚDE, 2003).

No percentil 90, são enquadradas as regiões consideradas de transmissão moderada. A incidência de LV nestes municípios variou de 8,2 a 9,1. Este estrato engloba as seguintes localidades, com suas respectivas incidências: Verdelândia $(8,2)$, Capitão Enéas $(8,8)$, Matias Cardoso $(9,1)$ e Porteirinha $(9,1)$. Já aquelas regiões com transmissão intensa obtiveram incidências que variaram de 9,5 a 15,2, sendo compostas pelas seguintes cidades e respectivas incidências: Francisco Sá (9,5), Janaúba (9,5), Santa Fé de Minas (10,3), Varzelândia $(13,6)$ e Grão Mogol $(15,2)$. Estes dois últimos municípios, Varzelândia com 13,6 e Grão Mogol com 15,2, apresentaram taxas muito elevadas de incidência, caracterizando bem o problema da LV na Mesorregião Norte de Minas.

Tabela 2 - Estratificação dos municípios segundo percentis - 1998/2002

\begin{tabular}{lcccccc}
\hline Percentil & $\begin{array}{c}\text { Limite } \\
\text { superior }\end{array}$ & $\begin{array}{c}\mathrm{N}^{\mathbf{o}} \text { de } \\
\text { municípios }\end{array}$ & Percentual & $\begin{array}{c}\text { Percentual } \\
\text { acumulado }\end{array}$ & $\begin{array}{c}\text { Percentual do } \\
\text { total de mun. }\end{array}$ & Transmissão \\
\hline$[10 ; 40]$ & 3,2 & 18 & 40,0 & 40,0 & 20,2 & Esporádica \\
50 & 3,9 & 5 & 11,1 & 51,1 & 5,6 & Esporádica \\
60 & 4,9 & 4 & 8,9 & 60,0 & 4,5 & Esporádica \\
70 & 6,1 & 4 & 8,9 & 68,9 & 4,5 & Esporádica \\
80 & 8,1 & 5 & 11,1 & 80,0 & 5,6 & Esporádica \\
90 & 9,4 & 4 & 8,9 & 88,9 & 4,5 & Moderada \\
$>90$ & 15,2 & 5 & 11,1 & 100,0 & 5,6 & Intensa \\
Total & - & 45 & 100 & - & 50,6 & - \\
\hline
\end{tabular}

Fonte: Resultados da pesquisa. 
A fim de facilitar a visualização da incidência da zoonose na Região, a Figura 1 (a) mostra sua distribuição espacial segundo a estratificação da tabela anterior no período 1998/2002. Uma primeira análise deste mapa indica que, apesar de existirem áreas com incidência nula no quinquênio, provavelmente não existe área livre de risco nesta região de Minas Gerais. Todo município é contíguo a outro com casos de LV, possuem fluxo migratório intenso entre eles e,ou fazem parte do mesmo eixo rodoviário dos municípios com casos de LV, o que torna todos os municípios vulneráveis à doença.

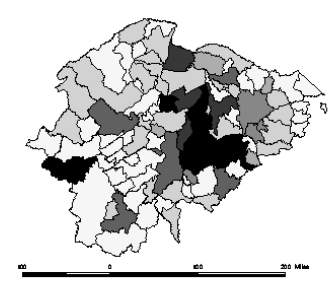

(a)

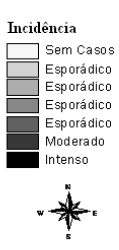

(b)

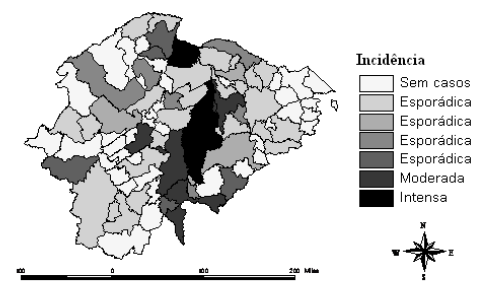

(b)

Figura 1: Distribuição espacial da LV no Norte de Minas entre 1998 e 2002 (a); e entre 2003 e 2008 (b).

Fonte: Resultados da Pesquisa.

Para analisar a dinâmica da doença até o ano de 2008, faz-se neste momento uma análise similar à anterior para o período de 2003/2008. As estatísticas descritivas desse caso estão na Tabela 3. Novamente, a média entre as duas amostras é muito diferente, assim como o coeficiente de variação. A incidência média de LV nos municípios com casos foi de 44, com CV de $119 \%$.

Tabela 3 - Estatísticas descritivas da incidência de LV no período de 2003/2008

\begin{tabular}{ccccc}
\hline Amostra & Classe & Média & Desvio padrão & CV (\%) \\
\hline $\mathrm{n}=89$ & Todos os municípios & 24 & 45 & 183 \\
$\mathrm{n}=49$ & Municípios com casos & 44 & 53 & 119 \\
\hline
\end{tabular}

Fonte: Resultados da pesquisa. 
Ao analisar conjuntamente as Tabelas 1 e 3, pode-se ver que a média da incidência nos anos mais recentes é $76 \%$ superior à obtida entre os anos de 1998/2002. Isso indica que o número de casos registrados tendeu a aumentar ao longo de todo período (1998/2008). Além disso, o número de municípios com casos aumentou de 45 para 49 neste mesmo período de tempo.

O desvio padrão da incidência da doença também aumentou nos anos mais recentes. Por exemplo, nos municípios com casos registrados, no primeiro período considerado (1998/2002), essa estatística obteve o valor de 17; já no segundo período (2003/2008), foi de 53, isto é, um aumento de cerca de três vezes. Disso decorre o aumento das estatísticas do coeficiente de variação no período de 2002 a 2008, pois, apesar de a média dos casos ter aumentado, a elevação do desvio padrão foi mais que proporcional. Isso se deve a um grande aumento nos casos de LV nos municípios de maior incidência. A título de ilustração, no Município de Francisco Sá, o número total de casos passou de 47 para 120; em Montes Claros, a incidência passou de 40 para 74; e em Matias Cardoso, o aumento da doença foi crítico, passando de 45 para 355 casos por cem mil habitantes. Por si só, esses indicadores de incidência da LV mostram a gravidade crescente da doença na região, indicando a necessidade de se atuar com maior eficácia sobre seus determinantes sociais.

A Tabela 4 mostra a estratificação dos municípios segundo as taxas de incidência. Percebe-se que o limite superior dos percentis para todas as classes se elevou consideravelmente no segundo período comparativamente ao primeiro. Entre 2003/2008, 50\% dos municípios com casos de LV apresentaram 5,6 casos ou menos por cem mil habitantes, enquanto, aproximadamente, 80\% deles apresentaram 10,2 casos ou menos, considerados pela metodologia da Secretaria de Vigilância Sanitária de transmissão esporádica. Mas essa taxa de incidência, ao contrário de desprezível, é bastante elevada para o contexto nacional.

Os municípios com transmissão moderada, com taxa de incidência variando de 11 a 12,3, são os seguintes: Porteirinha $(11,0)$, Nova 
Porteirinha (11,1), Bocaiúva (11,4), Brasília de Minas $(11,6)$ e Montes Claros $(12,3)$. Com exceção de Porteirinha, todos os municípios constantes nesta categoria eram de transmissão esporádica entre 1998 e 2002.

Tabela 4 - Estratificação dos municípios segundo percentis - 2003/2008

\begin{tabular}{lcccccc}
\hline Percentil & $\begin{array}{c}\text { Limite } \\
\text { superior }\end{array}$ & $\begin{array}{c}\mathrm{N}^{\mathbf{o}} \text { de } \\
\text { municípios }\end{array}$ & Percentual & $\begin{array}{c}\text { Percentual } \\
\text { acumulado }\end{array}$ & $\begin{array}{c}\text { Percentual do } \\
\text { total de mun. }\end{array}$ & Transmissão \\
\hline$[10 ; 40]$ & 4,3 & 20 & 40,8 & 40,8 & 22,5 & Esporádica \\
50 & 5,6 & 5 & 10,2 & 51,0 & 5,6 & Esporádica \\
60 & 6,7 & 4 & 8,2 & 59,2 & 4,5 & Esporádica \\
70 & 7,2 & 5 & 10,2 & 69,4 & 5,6 & Esporádica \\
80 & 10,2 & 5 & 10,2 & 79,6 & 5,6 & Esporádica \\
90 & 12,4 & 5 & 10,2 & 89,8 & 5,6 & Moderada \\
$>90$ & 59,2 & 5 & 10,2 & 100 & 5,6 & Intensa \\
Total & - & 49 & 100 & - & 55.1 & - \\
\hline
\end{tabular}

Fonte: Resultados da pesquisa.

Os casos de LV das áreas consideradas de transmissão intensa aumentaram consideravelmente entre os períodos analisados. O limite superior do percentil P90 passou de 9,4 para 12,4, um crescimento de $32 \%$, aproximadamente. Os municípios constantes no intervalo equivalente ao último percentil de incidência, entre 2003 e 2008, foram: Juramento $(12,7)$, Capitão Enéas (16,8), Janaúba (18,1), Francisco Sá (20,0) e Matias Cardoso (59,2). A incidência de LV em Matias Cardoso entre 1998/2002 e 2003/2008 aumentou muito, cerca de 550\%. Tal município deve ser estudado pormenorizadamente pelo grande aumento nos casos de LV neste período.

Em geral, houve uma tendência de aumento nos casos de LV na mesorregião Norte de Minas. Isso pode ser verificado pela correlação de Spearman entre as incidências do primeiro e do segundo período de tempo. O coeficiente associação linear obteve o valor de 52\%, com o pvalor de 0,0 . Ou seja, os municípios que apresentaram muitos casos da LV no período de 1998/2002 tenderam a ser aqueles que tiveram muitos casos no período pós 2003. A distribuição espacial da LV no período de 2003/2008 está ilustrada na Figura 1, parte b. Esta figura mostra que, entre as áreas sem casos da doença, a maioria é vulnerável à LV. 
Uma primeira conclusão geral da análise anterior é que neste período não houve tendência para a diminuição de casos de LV na região. Tal fato sugere a necessidade de políticas públicas mais efetivas. As próximas análises realizadas procuram, assim, fornecer instrumentos pelos quais as administrações públicas podem atuar no sentido de diminuir os casos de LV no Norte de Minas. Para isso, em primeiro lugar, foram agrupados os municípios segundo a média da incidência de LV nos dois períodos considerados, para, em seguida, realizar a análise discriminante dos agrupamentos.

\subsection{Agrupamentos e discriminação dos municípios no Norte de Minas quanto à incidência de Leishmaniose Visceral}

Nesta etapa do trabalho, os municípios da região do Norte de Minas foram divididos quanto à incidência de LV nos períodos de 1998/2002 e 2003/2008, segundo a análise de clusters, ou agrupamentos. Esta análise, de acordo com Mingoti (2007), tem como objetivo dividir os elementos da amostra ou população em grupos de forma que os elementos similares pertençam ao mesmo grupo. Foram escolhidos três agrupamentos, divisão que melhor atendeu aos dois critérios anteriormente citados. O nível de fusão para esta divisão, que é uma medida de associação dos objetos, foi de cinco, um valor razoavelmente baixo quando comparado com os demais.

Um primeiro resultado interessante da análise de agrupamentos é que a cidade de Matias Cardoso foi mantida, com exceção do caso de dois agrupamentos, como um cluster isolado, isto é, tal localidade apresentou grande dissimilaridade em relação a todos os outros municípios. A Tabela 5 mostra os municípios segundo o grupo de pertinência. Pode-se ver que o número de áreas envolvidas em cada agrupamento é muito diferente. O grupo 1, por exemplo, tem pouco mais de $84 \%$ dos municípios, já o grupo 3 é constituído por apenas uma localidade, Matias Cardoso, o que equivale a pouco mais de $1 \%$ do total de municípios. A caracterização dos grupos feita a seguir se mostrou elucidativa. 
Tabela 5 - Agrupamentos e municípios englobados*

\begin{tabular}{|c|c|}
\hline Grupo & Municípios \\
\hline $\begin{array}{c}1 \\
(n=75)\end{array}$ & $\begin{array}{l}\text { Águas Vermelhas, Berizal, Bocaiúva, Bonito de Minas, Brasília de Minas, Buritizeiro, } \\
\text { Campo Azul, Catuti, Chapada Gaúcha, Claro dos Poções, Cônego Marinho, Coração de } \\
\text { Jesus, Cristália, Curral de Dentro, Divisa Alegre, Engenheiro Navarro, Espinosa, } \\
\text { Francisco Dumont, Fruta de Leite, Gameleiras, Glaucilândia, Guaraciama, Ibiaí, } \\
\text { lbiracatu, Icaraí de Minas, Itacambira, Itacarambi, Jaíba, Januária, Japonvar, Jequitaí, } \\
\text { Josenópolis, Juramento, Juvenília, Lagoa dos Patos, Lassance, Lontra, Luislândia, } \\
\text { Mamonas, Manga, Mato Verde, Mirabela, Miravânia, Montalvânia, Montezuma, } \\
\text { Ninheira, Nova Porteirinha, Novorizonte, Olhos-d'água, Padre Carvalho, Pai Pedro, } \\
\text { Patis, Pedras de Maria da Cruz, Pintópolis, Pirapora, Ponto Chique, Riachinho, Riacho } \\
\text { dos Machados, Rio Pardo de Minas, Rubelita, Salinas, Santa Cruz de Salinas, Santo } \\
\text { Antônio do Retiro, São João da Lagoa, São João da Ponte, São João das Missões, São } \\
\text { João do Pacuí, São João do Paraíso, São Romão, Serranópolis de Minas, Taiobeiras, } \\
\text { Ubaí, Urucuia, Vargem Grande do Rio Pardo }\end{array}$ \\
\hline $\begin{array}{c}2 \\
(n=13)\end{array}$ & $\begin{array}{l}\text { Botumirim, Capitão Éneas, Fracisco Sá, Grão Mogol, Janaúba, Monte Azul, Montes } \\
\text { Claros, Porteirinha, Santa Fé de Minas, São Francisco, Várzea da Palma, Varzelândia, } \\
\text { Verdelândia }\end{array}$ \\
\hline $\begin{array}{c}3 \\
(\mathrm{n}=1)\end{array}$ & Matias Cardoso \\
\hline
\end{tabular}

Fonte: Resultados da Pesquisa. Nota $(*)$ : Número de municípios entre parênteses.

De acordo com a Tabela 6, percebem-se dois tipos de agrupamentos distintos: um tipo de alta incidência de LV nos dois períodos; e outro de baixa incidência. Os grupos 2 e 3 são do primeiro tipo. O cluster 2 manteve uma alta taxa de incidência média de LV nos dois períodos de tempo e pode ser classificado de incidência intensa, apesar de ter sido classificado de transmissão esporádica entre 2003 e 2008. O grupo 3 passou de moderado a intenso nestes períodos, tendo sido o que apresentou maior número de casos em todo os anos considerados.

Já o grupo 1 é do segundo tipo. Esse cluster se manteve no primeiro percentil entre 1998 e 2002 e no $2^{\circ}$ percentil entre 2003 e 2008, com uma média de incidência baixa, o que é um ponto positivo já que este cluster é o que engloba maior número de municípios. Para facilitar a visualização dos grupos, sua distribuição espacial é ilustrada na Figura 2. 
Tabela 6 - Caracterização dos Agrupamentos de incidência de LV

\begin{tabular}{cccc}
\hline Indicador & \multicolumn{3}{c}{ Grupo } \\
& 1 & 2 & 3 \\
\hline Média LV 98/02 & 1,3 & 9,3 & 9,1 \\
Média LV 03/08 & 2,4 & 9,7 & 59,2 \\
Classificação em 98/02 & $1^{\circ}$ percentil & $10^{\circ}$ percentil & $9^{\circ}$ percentil \\
& (esporádica) & (intensa) & (moderada) \\
Classificação em 03/08 & $2^{\circ}$ percentil & $8^{\circ}$ percentil & $10^{\circ}$ percentil \\
& (esporádica) & (esporádica) & (intensa) \\
\hline
\end{tabular}

Fonte: Resultados da pesquisa.

Passa-se, agora, à análise das variáveis que podem discriminar os agrupamentos gerados anteriormente (Tabela 7). Esta tabela traz os coeficientes padronizados das duas funções discriminantes canônicas estimadas. Assim, nas entradas de cada célula desta tabela os coeficientes das variáveis podem ser diretamente comparados, ou seja, aquele indicador que possuir maior valor absoluto na função tem maior poder discriminatório dos municípios.

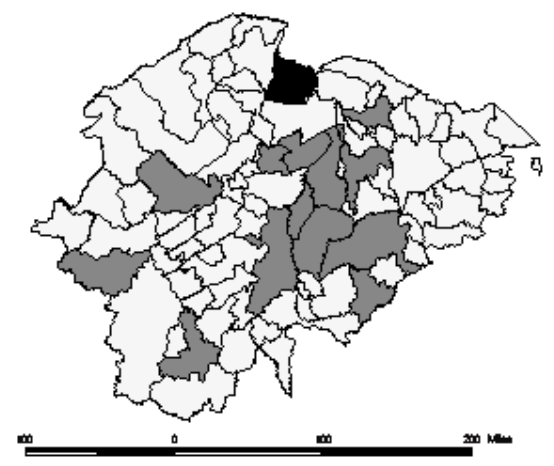

\section{Grupos:}

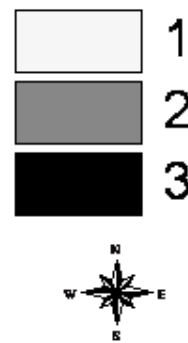

Figura 2: Distribuição espacial dos agrupamentos de incidências de Leishmaniose Visceral na mesorregião Norte de Minas.

Fonte: Resultados da pesquisa. 
Primeiramente, observa-se que dos 20 indicadores socioeconômicos utilizados de início, 11 foram selecionados pelo método stepwise. Além disso, o teste $\mathrm{M}$ de Box obteve um valor da estatística $\mathrm{F}$ de 4,4 com pvalor significativo a 1\%, dessa forma, a matriz de covariâncias dos grupos é estatisticamente diferente. O teste lambda de Wilks obteve uma estatística F de 2,8, significativa a $1 \%$, indicando que os vetores de médias dos grupos também são diferentes em cada variável considerada na análise discriminante. Por fim, cabe destacar que foram obtidas duas funções discriminantes: a primeira explica $57,5 \%$ da variância total entre os grupos, e a segunda, os $42,5 \%$ restantes.

Ao analisar a primeira função discriminante, percebe-se que existem variáveis discriminantes relacionadas ao perfil populacional dos municípios, como sua urbanização; à saúde, como os gastos com o item e a taxa de atendimento às crianças; à educação, como os gastos com o item e o percentual de atraso no ensino fundamental; e ao saneamento básico e à renda, desde que a qualidade das residências esteja relacionada com esta mesma renda.

Além disso, vê-se que variáveis relacionadas ao nível da urbanização das cidades, como a parcela de consumo de energia elétrica da população rural e o consumo residencial, bem como a própria taxa de urbanização, têm um grande poder discriminatório sobre os grupos de LV considerados. Isso pode ser reflexo de um efeito advindo do crescimento desordenado das cidades, que altera o meio ambiente natural e facilita a propagação dos mosquitos. Em seguida, tem-se o indicador de cobertura de água encanada e banheiro. $\mathrm{O}$ fato de esta variável estar relacionada às condições sanitárias dos municípios, como também acontece com a taxa de coleta de lixo, mostrou ser um resultado interessante, pois indica que medidas de limpeza urbana e eliminação dos resíduos sólidos orgânicos em locais inadequados, aliadas à eliminação de fontes de umidade e ao maior acesso à água encanada, são políticas capazes de evitar ou reduzir a proliferação do vetor com eficácia. 
Com respeito às variáveis de saúde, o principal indicador de discriminação são os gastos per capita com o item, o que é um ponto positivo, indicando que os gastos municipais com campanhas de prevenção e ataque direto ao vetor podem ser significativos na redução de casos de LV na Mesorregião Norte de Minas. Além disso, a taxa de atendimento a crianças de 7 a 17 também é importante, mas deve estar relacionada ao diagnóstico precoce da doença.

Tabela 7 - Resultados da análise discriminante, coeficientes padronizados nas funções discriminantes canônicas

\begin{tabular}{lcc}
\hline \multicolumn{1}{c}{ Variável } & \multicolumn{2}{c}{ Coeficiente na função } \\
& $\mathbf{1}$ & $\mathbf{2}$ \\
\hline Participação do consumo rural no consumo total de energia elétrica (\%) & 1,00 & $0,08^{*}$ \\
Pessoas que vivem em domić́lios com água encanada e banheiro (\%) & $-0,69^{*}$ & 0,33 \\
Taxa de urbanização & $0,68^{*}$ & 0,18 \\
Gasto per capita com saúde (R de 2004) & $0,57^{*}$ & 0,15 \\
Participação do consumo residencial no consumo total de energia & 0,45 & $0,99^{*}$ \\
elétrica (\%) & $-0,43^{*}$ & $-0,53$ \\
Percentual de pessoas que vivem em domicílios subnormais & $0,41^{*}$ & 0,11 \\
Percentual da população de 15 anos ou mais no total de matriculados no & $-0,35^{*}$ & 0,33 \\
fundamental & $-0,30$ & $0,29^{*}$ \\
Gasto per capita com educação (R de 2004) & 0,23 & $-0,42^{*}$ \\
Taxa de crescimento da população rural (1991/2000) - \% a. a. & $0,03^{*}$ & 0,43 \\
Taxa de atendimento de crianças e adolescentes de 7 a 17 anos $(\%)$ & \\
Pessoas que vivem em domicílios urbanos com coleta de lixo (\%) & \\
\hline
\end{tabular}

Fonte: Resultados da pesquisa. Nota (*): O asterisco representa a função discriminante em que a variável possui maior correlação em valor absoluto.

A educação também é importante. A significância dos gastos per capita com educação e do percentual da população de 15 anos ou mais no total de matriculados no fundamental (um indicador de atraso escolar) mostra que uma população mais bem instruída e capacitada é importante na diminuição de casos de LV, muito provavelmente em função do potencial caráter preventivo que a educação possui, além do melhor acesso à saúde que ela proporciona.

Por fim, tem-se o percentual de pessoas que vivem em domicílios subnormais, o sexto maior discriminante. Essa variável mede o percentual de indivíduos que vivem em aglomerações subnormais, como ocupações 
desordenadas ou ausência da titularidade da posse das propriedades. Estas são as características das habitações situadas em locais de baixa renda e que estão propícias a todo tipo de más condições sanitárias. Novamente, tem-se um indicador que encontra forte base na teoria.

Assim, de acordo com a hipótese levantada, foram encontradas variáveis de diversas áreas determinando a incidência de casos de LV na região. As medidas públicas para prevenção desta doença, portanto, não devem limitar-se às políticas de saúde, sendo as políticas educacionais, de saneamento básico, de regularização de loteamentos e de melhoria do bem-estar da população também importantes nesse sentido, isto é, os resultados indicam a aplicabilidade da teoria dos Determinantes Sociais da Saúde ao caso da leishmaniose visceral no Norte de Minas.

A análise implementada anteriormente mostrou-se bem ajustada. $\mathrm{O}$ índice global de acerto foi de aproximadamente $90 \%$ dos casos. Especificamente, 74 dos 75 casos do grupo 1 foram corretamente classificados; o grupo 3, composto por Matias Cardoso, foi bem classificado; e o grupo 2 obteve $31 \%$ de acerto, tendo sido sua classificação incorreta em relação ao do grupo 1 em localidades que não se diferenciavam consistentemente umas das outras.

\section{Conclusões}

A presente pesquisa sobre os determinantes sociais da Leishmaniose Visceral obteve resultados significativos que demandam a ação de políticas públicas. Os indicadores mais importantes na determinação do número de casos da doença foram o grau de urbanização e seu impacto sobre o meio ambiente natural do mosquito bem como as condições de saneamento básico. Assim, medidas simples, como limpeza urbana, eliminação de resíduos sólidos orgânicos e disponibilização de água encanada para a maior parcela da população, foram identificadas como iniciativas importantes para reduzir a proliferação da endemia. 
Ao analisar os indicadores relacionados à saúde, observou-se que a variável com maior poder de discriminação foi relacionada a gastos per capita nessa área, o que é um ponto positivo, pois indica que os gastos municipais com campanhas de prevenção e combate direto ao vetor podem ser significativos na redução de casos de LV na Mesorregião Norte de Minas. A educação também se mostrou um forte aliado ao combate da doença nesta região.

Em suma, conclui-se com o presente estudo que as ações de saúde pública necessárias a um maior controle da doença na região passam por políticas de sensibilização continuadas da população sobre a importância do combate à Leishmaniose Visceral, incluindo os cuidados que se deve ter com os animais domésticos, principais transmissores da doença, consubstanciadas com melhorias de saneamento básico e respeito ao meio ambiente do inseto.

\section{Referências}

ALENCAR, J. E.; NEVES, J. e DIETZE, R. Leishmaniose visceral. In: Doenças Infecciosas e Parasitárias (R. Veronesi, org.), pp. 706-717, São Paulo: Editora Guanabara Koogan, 1991.

ANDERSON, T.W. An introduction to multivariate statistical analysis. 3.ed. New York: John Wiley, 2003.

CANELA, J. R.; ALVES, C. J. M.; RODRIGUES, G. C. Perfil diagnóstico de Leishmaniose visceral em pacientes adultos admitidos no Hospital Universitário Clemente de Farias. Unimontes Científica, Montes Claros, v.6, n.2-jul/dez, 2004.

CHAVES, K. M. Estudo Dirigido sobre as Leishmanioses. Ministério da Saúde, Fundação Nacional de Saúde, Coordenação de Minas Gerais. Belo Horizonte, Janeiro de 1996. 
DAhlgren, G., WhITEHEAD, M. Policies and strategies to promote social equity in health. Stockholm: Institute for Futures Studies, 1991.

FERNAU, M.E; SAMSON, P.J. Use of cluster analysis to define periods of similar meteorology and precipitation hemistry in Eastern North America. Part I: Transport patterns. Journal of Applied Meteorology, Michigan, v.29, p.735-761, 1990.

FUNDAÇÃO JOÃO PINHEIRO. Índice Mineiro de Responsabilidade Social, 2009.

GONTIJO, C. M. F.; MELO, M. N. Leishmaniose visceral no Brasil: quadro atual, desafios e perspectivas. Revista Brasileira de Epidemiologia, São Paulo, v.7, n.3, p.338-349, 2004.

JOHNSON, D. E. Applied multivariate methods for data analysis. New York: Duxbury Press, 1998.

MingOTI, S. A. Análise de dados através de métodos de estatística multivariada: uma abordagem aplicada. Belo Horizonte: Editora UFMG, 2007.

MINISTÉRIO DA SAÚDE. Secretaria de Vigilância em Saúde. Departamento de Vigilância Epidemiológica. Manual de vigilância e controle da leishmaniose visceral. Série A, Normas e Manuais Técnicos, Brasília, 2003.

MIRANDA, G. M. D. Leishmaniose visceral em Pernambuco: a influência da urbanização e da desigualdade social. Dissertação (Mestrado em Saúde Pública) - Centro de Pesquisas Aggeu Magalhães, Fundação Oswaldo Cruz, 2008.

MONTEIRO, E. M. et al. Leishmaniose visceral: estudo de flebotomíneos e infecção canina em Montes Claros, Minas Gerais. Revista da Sociedade Brasileira de Medicina Tropical, vol. 32, n.2, p. 147-152, mar-abr, 2005. 
PEDROSA C. M. S; ROCHA, E. M. M. Aspectos clínicos e epidemiológicos da leishmaniose visceral em menores de 15 anos procedentes de Alagoas, Brasil. Revista da Sociedade Brasileira de Medicina Tropical, v.37, p.300-304, 2004.

POSSOLI, S. Técnicas de análise multivariada para avaliação das condições de saúde dos municípios do Rio Grande do Sul, Brasil. Revista de Saúde pública. São Paulo, 18: 288 - 300, 1984.

SOUSA, R. G et al. Casos de Leishmaniose Visceral Registrados no Município de Montes Claros, Estado de Minas Gerais. Acta Sci. Health SCI. MARINGÁ, V. 30, N.2. P. 155-159, 2008.

TAVARES L. M. S. A; TAVARES, E. D. Incidência, distribuição geográfica e aspectos ambientais das áreas endêmicas da leishmaniose visceral do Sergipe. Informe Epidemiológico do Sistema Único de Saúde n.8, p.47-52, 1999. 
REVISTA DE ECONOMIA E AGRONEGÓCIO, VOL.9, $N^{\circ} 1$

\section{Anexos}

Tabela 1.A - Indicadores utilizados na análise discriminante

Fonte: Fundação João Pinheiro (FJP) e Instituto Brasileiro de Geografia e Estatística (IBGE).

Variável

Rendimento $n$

Participação d

Participação d

Percentual de

Percentual da

Taxa de aten

Percentual da

fundamental

Gasto per cap

Percentual de

doenças e agr

Gasto per cap

$\%$ de pessoas

$\%$ de pessoas

Percentual de

Percentual de

Percentual de

Percentual de por dormitóri

Taxa de cresc

Taxa de cresci

Taxa de cresci

Taxa de urban 